\title{
NON SINGULAR HAMILTONIAN SYSTEMS AND GEODESIC FLOWS ON SURFACES WITH NEGATIVE CURVATURE
}

\author{
Ernesto A. Lacomba and J. Guadalupe Reyes
}

\begin{abstract}
We extend here results for escapes in any given direction of the configuration space of a mechanical system with a non singular bounded at infinity homogeneus potential of degree -1 , when the energy is positive. We use geometrical methods for analyzing the parallel and asymptotic escapes of this type of systems. By using Riemannian geometry methods we prove under suitable conditions on the potential that all the orbits escaping in a given direction are asymptotically parallel among themselves. We introduce a conformal Riemannian metric with negative curvature in the interior of the Hill's region for a fixed positive energy level and we consider the boundary as a singular part of the infinity. The associated geodesic flow has as solution curves those of the problem for a fixed energy. We perform the compactification of the region via the limiting directions of the geodesic flow, obtaining a closed unit disk with a quasi-complete metric of negative curvature.
\end{abstract}

\section{Introduction}

By a classical mechanical system we understand a triple $(M, g, U)$ where $M$ is a Riemannian manifold with metric $g$, the function $K$ : $T M \rightarrow \mathbb{R}$ defined by $K_{x}(v)=\frac{1}{2} g(v, v)$ for all $v \in T_{x} M$ is called the kinetic energy of the system, and $U: M \rightarrow \mathbb{R}$ is a smooth function defined in $M$ called the potential of the system. We note that $K$ is essentially the square of the norm of the Riemannian metric, when applied to tangent vectors of the manifold $M$.

The function $E(x, v)=K_{x}(v)+U(\pi(v))$ is called the total energy of the system, with $x \in M$ and $v \in T_{x} M$, which means $x=\pi(v)$, where $\pi: T M \rightarrow M$ is the canonical projection. The variable $v$ is known as the velocity.

This work was partially supported by CONACYT grants with numbers 1772-E9210 and 400200-5-1406PE. 
If the Riemannian metric in local coordinates is given by $g=\left(g_{i j}\right)$ (a symmetric positive definite matrix), then, when we consider the local kinetic energy $K(x, v)=\frac{1}{2} g_{i j} v^{i} v^{j}$, the total energy in these local coordinates $(x, v)$ is written $E(x, v)=\frac{1}{2} g_{i j} v^{i} v^{j}+U(x)$. From now on we adopt the summation convention on the repeated indices in a given formula.

The physical curves of a classical mechanical system are the extremal curves of the variational principle corresponding to the Lagrangian $L$ : $T M \rightarrow \mathbb{R}$ defined as

$$
L(x, v)=K_{x}(v)-U(\pi(v)) .
$$

In coordinates $L=L(x, v)$ the Euler-Lagrange equations for the variational principle are written

$$
\frac{d}{d t}\left(\frac{\partial L}{\partial v^{k}}\right)=\frac{\partial L}{\partial x^{k}}, \quad \frac{d x^{k}}{d t}=v^{k}
$$

In a neighborhood of the point $(x, p) \in T^{*} M$, we can construct a diffeomorphism $T^{*} M \rightarrow T M$ locally defined by $\left(x^{i}, p_{i}\right) \rightarrow\left(x^{i}, g^{i j} p_{j}\right)=$ $\left(x^{i}, v^{i}\right)$. This diffeomorphism carries the total energy function $E(x, v)$ into a function $\mathcal{H}: T^{*} M \rightarrow \mathbb{R}$ locally defined by $\mathcal{H}(x, p)=\frac{1}{2} g^{i j} p_{i} p_{j}+$ $U(x)$, and called the Hamiltonian function. The function defined locally by $v^{i}=g^{i j} p_{j}$ is called the Legendre Transformation.

The Legendre transformation carries the physical curves into $T^{*} M$. Those curves in local coordinates of $T^{*} M$ are the solutions of the following system of first order diferential equations:

$$
\left\{\begin{array}{l}
\dot{x}^{i}=\frac{\partial \mathcal{H}}{\partial p_{i}} \\
\dot{p}_{i}=\frac{-\partial \mathcal{H}}{\partial x^{i}}
\end{array}\right.
$$

known as the Hamilton equations.

If $(x, p)=(x(t), p(t))$ is a solution curve of $(2)$ in $T^{*} M$, we have that $\frac{d \mathcal{H}}{d t}(x(t), p(t))=\frac{\partial \mathcal{H}}{\partial x} \dot{x}+\frac{\partial \mathcal{H}}{\partial p} \dot{p}=0$, and from this we have that the Hamiltonian $\mathcal{H}$ is constant along the solutions of (2). This is the principle of conservation of energy which shows that it is sufficient to consider the system for fixed levels of energy. Since the Legendre transformation is a diffeomorphism, the total energy $E$ is also a constant of motion for the physical curves or solutions of (1) in $T M$. In this way, we split the total system into systems defined on the energy surfaces $\mathcal{H}=h$ lying in the phase space $T^{*} M$, with an effective reduction in one dimension. 
We note that the Hamiltonian system could be locally considered as defined in the tangent bundle $T M$ instead of the cotangent bundle $T^{*} M$, because of the Legendre transformation.

For the fixed energy level $h$, in local coordinates we obtain the energy equation $h=\frac{1}{2} g^{i j} p_{i} p_{j}+U(x)$. Since $g^{i j} p_{i} p_{j} \geq 0$ the energy relation define the set

$$
M_{h}=\{x \in M \mid U(x) \leq h\},
$$

the so-called Hill's region of system for a fixed energy level $h$ and it is the set of possible permissible configurations for this level. We assume here that $M_{h}$ is a connected, simply connected subset of $M$.

The boundary curve $\Gamma=\left\{x \in M_{h} \mid U(x)=h\right\}$ is where the kinetic energy vanishes and is called the zero velocity manifold.

By Sard's Theorem, except for a set of values $h$ with Lebesgue measure zero, we can suppose $M_{h}$ is a submanifold of $M$ with boundary $\Gamma$, which we assume to be also connected.

\subsection{Geodesic flows.}

When we consider a system $(M, g, U)$ such that $U \equiv 0$, the corresponding Hamiltonian has a total energy function which coincides with the Lagrangian and it is locally written $E=L=\frac{1}{2} g_{i j} v^{i} v^{j}$.

We have the variational problem

$$
0=\delta \int_{P}^{Q} g_{i j} \dot{x}^{i} \dot{x}^{j} d t=\delta \int_{P}^{Q}\|\dot{x}\|_{g}^{2} d t
$$

defined in the tangent bundle $T M$ on all the curves $\gamma: x=x(t)$ joining the points $P, Q \in M$. The extremal curves (which satisfy the EulerLagrange equations) will be called the geodesics in the space $M$ relative to the metric $g$, which in local coordinates is written $g_{i j}$.

The set of all the solutions of this variational problem is called the geodesic flow in the manifold $M$, and are locally those curves $x=x(t)$ solving the following system of differential equations

$$
\ddot{x}^{i}=-\Gamma_{j k}^{i} \dot{x}^{j} \dot{x}^{k}
$$

where $\Gamma_{j k}^{i}=\frac{1}{2} g^{i \ell}\left(\frac{\partial g_{\ell k}}{\partial x^{j}}+\frac{\partial g_{j \ell}}{\partial x^{k}}-\frac{\partial g_{j k}}{\partial x^{\ell}}\right)$ are the Christoffel numbers of the connection asociated to the metric $g_{i j}([\mathbf{D u}],[\mathbf{S p}])$.

We can think of the geodesic flow on a Riemannian manifold as the set of physical curves of the Hamiltonian system locally written as $\mathcal{H}=$ $\frac{1}{2} g^{i j} p_{i} p_{j}$ not subject to any potential. 


\subsection{Systems with two degrees of freedom.}

We are interested here in Hamiltonians in $\mathbb{R}^{2}$ of type

$$
\mathcal{H}=\frac{1}{2} g^{i j} p_{i} p_{j}+U(x, y)
$$

having two degrees of freedom and where $g_{i j}=\left(\begin{array}{cc}m_{1} & 0 \\ 0 & m_{2}\end{array}\right)$ is a mass matrix (positive definite), that is, in Hamiltonians whose energy function in the variables $(x, y, \dot{x}, \dot{y})$, have the form

$$
E=\frac{1}{2}\left(m_{1} \dot{x}^{2}+m_{2} \dot{y}^{2}\right)+U(x, y),
$$

where $U(x, y)$ is the potential of the system. To obtain from the Hamiltonian the energy function defined in the tangent bundle of $\mathbb{R}^{2}$, we use the Legendre transformation $g^{i j} p_{j}=\dot{x}^{i}$.

Without loss of generality, we can study the Hamiltonians such that the energy function in the variables $(x, y, \dot{x}, \dot{y})$ has the form

$$
E=\frac{1}{2}\left(\dot{x}^{2}+\dot{y}^{2}\right)+\tilde{U}(x, y)
$$

which is obtained from the above by a linear change of coordinates.

\section{Curvature of the Mechanical System}

We begin this section with the Maupertuis least action principle. The proof can be seen in the references $[\mathbf{D u}],[\mathbf{A r}]$.

Theorem 1 (Maupertuis). If our system (1) is autonomous, then for a fixed energy level $h$, its physical solutions are the extremals of the variational problem

$$
0=\delta \int_{\gamma} 2 K(\dot{\gamma}(t)) d t
$$

defined on all the curves $(\gamma(t), \dot{\gamma}(t)) \in T M_{h}$.

If in local coordinates $K(\dot{\gamma}(t))=\frac{1}{2} g_{i j} \dot{\gamma}^{i} \dot{\gamma}^{j}$ is the kinetic energy of the system, then the physical solutions are the extremals with fixed energy $h$ of the variational problem with Lagrangian $\tilde{L}=2\left(\frac{1}{2} g_{i j} \dot{x}^{i} \dot{x}^{j}\right)=\|\dot{x}\|_{g}^{2}$.

Corresponding to the metric $g_{i j}$ we have the Lagrangian $L=\sqrt{g_{i j} \dot{x}^{i} \dot{x}^{j}}$, whose corresponding functional defines arc length along curves.

Its extremals are parameter independent curves, but the values of the functional on extremals permit to define the arc length parameter.

A simple computation gives the following standard result. 
Proposition 1 ([Du], $[\mathbf{S p}])$. The Lagrangian $\tilde{L}=g_{i j} \dot{x}^{i} \dot{x}^{j}$ has the same extremals as $L=\sqrt{g_{i j} \dot{x}^{i} \dot{x}^{j}}$ up to a reparametrization.

This implies that the geodesics are exactly those curves extremizing arc length.

We observe from Theorem 1 that for a fixed energy level $h$, it is sufficient to calculate the geodesic flow corresponding to the metric $g_{i j}$ for obtaining the physical motions of problem (1); from Proposition 1 it is sufficient with studying this flow as an one-dimensional foliation in $M_{h}$.

We consider now on $M=\mathbb{R}^{n}$ a Lagrangian of the form

$$
L=\frac{1}{2} \delta_{i j} \dot{x}^{i} \dot{x}^{j}-U(x),
$$

then by the Maupertuis principle we have that for a fixed energy level $h$, the extremals of the problem

$$
S(\gamma)=\int_{\gamma} \delta_{i j} \dot{x}^{i} \dot{x}^{j} d t, \quad \gamma \subset M_{h} \subset \mathbb{R}^{n}
$$

are the physical solutions of the mechanical problem

$$
\ddot{x}=-\nabla U(x) .
$$

On the interior $\operatorname{int}\left(M_{h}\right)$ of the Hill's region the identity $1=\frac{\delta_{i} \dot{x}^{i} \dot{x}^{j}}{2(h-U)}$ holds. In particular if the curve $\gamma$ does not touch the boundary $\Gamma$ of Hill's region, it is always valid. Hence

$$
S(\gamma)=\int_{\gamma} \delta_{i j} \dot{x}^{i} \dot{x}^{j}=\int_{\gamma} \frac{\left(\delta_{i j} \dot{x}^{i} \dot{x}^{j}\right)^{2}}{2(h-U)}=\int_{\gamma} \frac{1}{2} \frac{\left(\delta_{i j} \dot{x}^{i} \dot{x}^{j}\right)^{2}}{(h-U)} .
$$

From Proposition 1, the Lagrangians

$$
\tilde{L}=\delta_{i j} \dot{x}^{i} \dot{x}^{j}=\frac{1}{2} \frac{\left(\delta_{i j} \dot{x}^{i} \dot{x}^{j}\right)^{2}}{(h-U)}
$$

and

$$
L=\frac{1}{\sqrt{2}} \frac{\delta_{i j} \dot{x}^{i} \dot{x}^{j}}{\sqrt{h-U}}
$$

have the same extremals in the interior of the Hill's region.

In this way, we introduce the metric $g_{h}$ in the interior of the Hill's region $M_{h}$ locally defined by

$$
\left(g_{h}\right)_{i j}=\left(\sqrt{2} \frac{\delta_{i j}}{\sqrt{h-U}}\right)
$$


whose geodesic flow in the interior is related to the physical curves of problem (3) and their corresponding arc length parameter is $\ell$, where $\frac{d \ell}{d t}=\left(\frac{2}{h-U}\right)^{1 / 4}\|d \gamma\|$ is a reparametrization of physical time, where $\|d \gamma\|^{2}=\dot{x}^{2}+\dot{y}^{2}$.

With this we obtain the

Proposition 2. For a fixed energy level h, the physical curves of the system (3) are the geodesics asociated to the Riemannian metric $\left(g_{h}\right)_{i j}=$ $\sqrt{2} \frac{\delta_{i j}}{\sqrt{h-U}}$ defined in $\operatorname{int}\left(M_{h}\right)$.

Proof: Let $\ell$ be the arc length as we defined before. Then $\frac{d \ell}{d t}=$ $\left(\frac{2}{h-U}\right)^{1 / 4} \sqrt{\delta_{i j} \dot{x}^{i} \dot{x}^{j}}$, which implies that $\left(\frac{d \ell}{d t}\right)^{2}=\frac{\sqrt{2}}{\sqrt{h-U}} \delta_{i j} \dot{x}^{i} \dot{x}^{j}$.

If we consider the Lagrangian associated to the metric $g_{h}$ :

$$
L=\frac{1}{2}\left(g_{h}\right)_{i j} \dot{x}^{i} \dot{x}^{j}=\frac{\sqrt{2}}{2 \sqrt{h-U}} \delta_{i j} \dot{x}^{i} \dot{x}^{j},
$$

and denote by $\left({ }^{\prime}\right)$ the derivative respect to $\ell$, we have that

$$
\begin{aligned}
L & =\frac{\sqrt{2}}{2} \frac{\delta_{i j}\left(x^{i}\right)^{\prime}\left(x^{j}\right)^{\prime}}{\sqrt{h-U}}\left(\frac{d \ell}{d t}\right)^{2} \\
& =\frac{2 \delta_{i j}\left(x^{i}\right)^{\prime}\left(x^{j}\right)^{\prime}}{2(h-U)}\left(\delta_{i j} \dot{x}^{i} \dot{x}^{j}\right) \\
& =2 \delta_{i j}\left(x^{i}\right)^{\prime}\left(x^{j}\right)^{\prime}
\end{aligned}
$$

In the last equality we used the fact that $\frac{\delta_{i j} \dot{x}^{i} \dot{x}^{j}}{2(h-U)}=1$ in the interior of the Hill's region.

A curve $x=x(\ell)$ is a geodesic for the metric $g_{h}$, if and only if it satisfies the Euler-Lagrange equations:

$$
\frac{d}{d \ell}\left(\frac{\partial L}{\partial\left(x^{k}\right)^{\prime}}\right)=\frac{\partial L}{\partial x^{k}}
$$

A straightforward calculation shows that on the curve $x=x(\ell)$ :

$$
\frac{\partial L}{\partial x^{k}}=0 \quad \text { and } \quad \frac{d}{d \ell}\left(\frac{\partial L}{\partial\left(x^{k}\right)^{\prime}}\right)=4 \delta_{k j}\left(x^{j}\right)^{\prime \prime}=4\left(x^{k}\right)^{\prime \prime} .
$$

This is equivalent to $\left(x^{k}\right)^{\prime \prime}=0$, which ends the proof. 
We say that the metric $g_{h}$ is the blowing up of the metric $\delta_{i j}$ on the zero velocity curve $\Gamma$, because it carries that boundary into part of the "infinity" of the Hill's region, but with a different behaviour from other points at infinity.

We define the curvature of the new mechanical system $\left(\operatorname{int}\left(M_{h}\right), g_{h}, U\right)$ as the Gaussian sectional curvature of the metric $g_{h}$. For the case of two variables $x^{1}=x, x^{2}=y$ we have the following result:

Theorem 2 (Curvature). Let $k(x, y)$ be the Gaussian curvature related to the metric $g_{h}$ at the point $(x, y)$ of the interior of Hill's region $M_{h}$. If $U(x, y)$ is a class $C^{2}$ potential, then, for the fixed level energy $h$ we have that

1. If $\Delta U(x, y)>-4\|\nabla \sqrt{h-U}\|^{2}$ then $k(x, y)<0$.

2. If $\Delta U(x, y)=-4\|\nabla \sqrt{h-U}\|^{2}$ then $k(x, y)=0$.

3. If $\Delta U(x, y)<-4\|\nabla \sqrt{h-U}\|^{2}$ then $k(x, y)>0$.

Here $\Delta=\frac{\partial}{\partial x^{2}}+\frac{\partial}{\partial y^{2}}$ is the Laplacian operator and $\nabla$ denotes the gradient.

Proof: We observe that $\left(g_{h}\right)_{i j}$ has the form $f(x, y) \delta_{i j}$, where

$$
f(x, y)=\frac{\sqrt{2}}{\sqrt{h-U(x, y)}},
$$

and therefore $g_{h}$ is conformal with the planar metric $\delta$. We use the Gauss formula (see $[\mathbf{D u}]$ ) for the curvature:

$$
k(x, y)=\frac{-1}{2 f(x, y)} \Delta \log f(x, y) .
$$

A straigthforward calculation gives us

and then

$$
\Delta \log f(x, y)=\frac{(h-U) \Delta U+\|\nabla U\|^{2}}{2(h-U)^{2}}
$$

$$
\begin{aligned}
k(x, y) & =\frac{-\sqrt{h-U}}{2 \sqrt{2}} \cdot\left[\frac{(h-U) \Delta U+\|\nabla U\|^{2}}{2(h-U)^{2}}\right] \\
& =\frac{-(h-U)^{3 / 2}}{4 \sqrt{2}(h-U)^{2}} \cdot\left[\Delta U+\left\|\frac{\nabla U}{\sqrt{h-U}}\right\|^{2}\right] \\
& =\frac{-1}{4 \sqrt{2} \sqrt{h-U}} \cdot\left[\Delta U+4\|\nabla \sqrt{h-U}\|^{2}\right] \\
& =\frac{-1}{4 \sqrt{2(h-U)}} \cdot\left[\Delta U+4\|\nabla \sqrt{h-U}\|^{2}\right],
\end{aligned}
$$

which ends the proof. 
Corollary 1. With the same conditions of the above theorem we have that

1. If $\Delta U(x, y)>0$ then $k(x, y)$ is negative.

2. If $\Delta U(x, y)=0$ and $\nabla U \neq 0$ then the curvature $k(x, y)$ is negative.

3. If $0>\Delta U>-\frac{\|\nabla U\|^{2}}{h-U}$ then the curvature $k(x, y)$ is negative.

Proof: From Theorem 2 we have that

$$
\begin{aligned}
k(x, y) & =\frac{-\sqrt{h-U}}{4 \sqrt{2}}\left[\frac{(h-U) \Delta U+\|\nabla U\|^{2}}{(h-U)^{2}}\right] \\
& =\frac{-1}{4 \sqrt{2(h-U)}}\left[\Delta U+\frac{\|\nabla U\|^{2}}{h-U}\right],
\end{aligned}
$$

and this proves the statement, because the quantity in the last parentheses is positive in each one of the three cases.

We consider now potentials $U: M_{h} \rightarrow \mathbb{R}$ not having singularities in the sense that some of the denominators vanish as in the case of collisions between particles in Celestial Mechanics. Then, for any initial condition for the system of differential equations (3), there exists a solution in the phase space whose projection in the Hill's region is an extremal $\gamma(t)=(x(t), y(t))$ defined in some interval $J \subset \mathbb{R}$. Here $t$ denotes a new parameter, instead of the physical time.

We say that a potential $U: M_{h} \rightarrow \mathbb{R}$ is bounded at infinity if there exist two positive numbers $R$ and $A$ such that for every $(x, y) \in M_{h} \backslash B_{R}(0,0)$ the inequality $|U(x, y)| \leq A$ holds, where $B_{R}(0,0)$ is the open ball of radius $R$ and center $(0,0)$.

The following result shows that all escaping geodesics have infinite arc length in the metric $g_{h}$.

Lemma 1. Let $U: M_{h} \rightarrow \mathbb{R}$ be a potential of class $C^{2}$, bounded at infinity, and let $\gamma$ be a solution of (3) in the Hill's region that escapes to infinity. Then, if long $(\gamma(t))$ is the arc length in the metric $g_{h}$ of curve $\gamma$ at time $t$, from the point $P_{0} \in \gamma$, we have that

$$
\lim _{t \rightarrow \infty} \operatorname{long}(\gamma(t))=\infty
$$


Proof: Let $A$ be a bound at infinity for the potential $U$, i.e., $|U(x, y)| \leq$ $A$ for all $(x, y)$ far enough from the origin.

From the chain of inequalities $0<|h-U| \leq|h|+|U| \leq h+A=M^{2}$ with $M>0$, we have that $0<\sqrt{h-U} \leq M$. This implies that

$$
\int_{P_{0}}^{\gamma(t)} \frac{\sqrt{d x^{2}+d y^{2}}}{\sqrt{M}} \leq \int_{P_{0}}^{\gamma(t)} \sqrt{\frac{d x^{2}+d y^{2}}{\sqrt{h-U}}}
$$

or equivalently $\frac{1}{\sqrt{M}} \operatorname{long}_{\delta}(\gamma(t)) \leq \operatorname{long}(\gamma(t))$, where $\operatorname{long}_{\delta}(\gamma(t))$ is the arc length of $\gamma$ from $P_{0}$ to $\gamma(t)$ in the Euclidean metric.

Since $\gamma(t)$ escapes, then $\lim _{t \rightarrow \infty} \operatorname{long}_{\delta}(\gamma(t))=\infty$, which implies necessarily that $\lim _{t \rightarrow \infty} \operatorname{long}(\gamma(t))=\infty$.

We now prove that the arc length in the metric $g_{h}$ of any geodesic going to the zero velocity curve $\Gamma$ is finite. This is related to the fact that the metric is singular in $\Gamma$, but the corresponding improper integral is convergent.

Lemma 2. Let $\gamma \subset M_{h}$ be a geodesic such that $\gamma$ intersects the zero velocity curve $\Gamma$ at the point $P_{0}$, and let $Q$ be an arbitrary point of $\gamma$ in the interior of the Hill's region. Then the arc length of $\gamma$ from $Q$ to $P_{0}$ is finite.

Proof: We consider the chain of equalities

$$
\begin{aligned}
\operatorname{long}\left(\gamma\left(P_{0}, Q\right)\right) & =\int_{P_{0}}^{Q} \sqrt{\frac{d x^{2}+d y^{2}}{\sqrt{h-U}}}=\int_{P_{0}}^{Q} \sqrt{\frac{\dot{x}^{2}+\dot{y}^{2}}{\sqrt{h-U}}} d t \\
& =\sqrt{2} \int_{P_{0}}^{Q} \frac{\sqrt{h-U}}{\sqrt[4]{h-U}} d t=\sqrt{2} \int_{P_{0}}^{Q} \sqrt[4]{h-U} d t .
\end{aligned}
$$

The last integral converges because there are no singularities.

From the last results we see that the geodesics through a point escaping to $\infty$ are defined for all values of the parameter in $\mathbb{R}^{+}$. On the other hand, those intersecting the zero velocity curve $\Gamma$ are defined only for values of the parameter bounded from above. We conclude the following:

Corollary 2. Let $U: M_{h} \rightarrow \mathbb{R}$ be a potential of class $C^{2}$, bounded at infinity, without singularities or critical points. Then the system (int $\left.\left(M_{h}\right), g_{h}, U\right)$ is not geodesically complete. 
We observe that the conditions on the potential in Corollary 2 are valid in the standard repulsive problems. For some examples see Section 5.

In Section 4 we will use these results for analyzing the global behaviour of the solutions of Hamiltonian systems with these properties.

We remark that Ong in $[\mathbf{O}]$ studied problems with central forces by using conformally Euclidean metrics (the so called Jacobi metrics) in a similar way than we did here. Such metrics have the property of vanishing in the zero velocity manifold of the Hill's region. Instead, the metric used in this section is not defined in such set, but geodesical distance to reach it is finite. Beginning with Section 4, we will consider suitable conditions on the potential, so that the curvature of the mechanical system is negative. The resulting geometry generalizes the behavior of the Kepler problem on positive energy surfaces, which is equivalent to the geodesic flow on the Poincare disk, whose curvature is constant and equal to -1 .

\section{Parallel escapes}

We use here, a blowing up of the "infinity" in the Hill's region, which can be called of inversion-projective type. Such blowing up has been used in [LR1] and [LR2] for analyzing parallel escapes to straight lines through the origin. In other words, we analyze the solutions to problem (3) in the Hill's region having a limiting slope at infinity. This sort of escape is possible for Hamiltonian systems whose potential function is homogeneous and when the energy is positive. Lacomba [L2] has shown that in a neighborhood of infinity on a positive energy surface there exists a foliation by submanifolds consisting of orbits escaping in each direction of the configuration space. We include here a new proof of this result, stated as Theorem 4 .

In order to use the methods of $[\mathbf{L R 1}]$ and [LR2] it is necessary to eliminate the physical time $t$ replacing it in a neighborhood of infinity by one of the configuration variables. This is true, provided that such variable has positive velocity at infinity. We generalize one result of [LR2] in the following lemma:

Lemma 3. Let $U=M_{h} \rightarrow \mathbb{R}$ be a potential with two degrees of freedom in the variables $(x, y)$, such that $\frac{\partial U}{\partial x} \neq 0$, and for certain initial conditions the coordinate $x=x(t)$ of the corresponding solution is defined for all physical time $t>0$ and $\dot{x}_{\infty}=\lim _{t \rightarrow \infty} \dot{x}(t)$ exists and is positive. Then $\lim _{t \rightarrow \infty} x(t)=\infty$. 
Proof:

1. If $\frac{\partial U}{\partial x}>0$, then from equation (3) in the two variables $x, y$ we have that $\ddot{x}(t)<0$, wich implies that $\dot{x}(t)$ is a decreasing function. By the Mean Value Theorem there exists $t^{*} \in\left(t_{0}, t\right)$ such that $x(t)-x\left(t_{0}\right)=\dot{x}\left(t^{*}\right)\left(t-t_{0}\right)$, and as $\dot{x}(t)$ is decreasing, then $x(t)-$ $x\left(t_{0}\right)=\dot{x}\left(t^{*}\right)\left(t-t_{0}\right)>\dot{x}(t)\left(t-t_{0}\right)$. Since $\lim _{t \rightarrow \infty} \dot{x}(t)=\dot{x}_{\infty}$ then $x(t)-x\left(t_{0}\right)>\dot{x}(t)\left(t-t_{0}\right) \geq \dot{x}_{\infty}\left(t-t_{0}\right)$. Since $\dot{x}_{\infty}>0$ then the claim is true for this case.

2. If $\frac{\partial U}{\partial x}<0$ then $\ddot{x}(t)>0$, which implies that $\dot{x}(t)$ is an increasing function. By the Mean Value Theorem there exists $t^{*} \in\left(t_{0}, t\right)$ such that $x(t)-x\left(t_{0}\right)=\dot{x}\left(t^{*}\right)\left(t-t_{0}\right)>\dot{x}\left(t_{0}\right)\left(t-t_{0}\right)$, that is, $x(t)>\dot{x}\left(t_{0}\right)\left(t-t_{0}\right)+x\left(t_{0}\right)$. Since $\lim _{t \rightarrow \infty} \dot{x}(t)=\dot{x}_{\infty}>0$, we can chose $t_{0}$ big enough such that $\dot{x}\left(t_{0}\right)>0$, which ends the proof.

In this way, if the partial derivative $\frac{\partial U}{\partial x}$ of the potential $U$ does not vanish, then for big enough times $t \in\left[t_{0}, \infty\right)$ we can replace the variable $t$ by the variable $x$. This means that we can consider the other configuration variable $y$ as a function of $x$, that is $y=y(x)$.

We have now the system of differential equations (3) in the case when there are only two degrees of freedom

$$
\left\{\begin{array}{l}
\ddot{x}=-\frac{\partial U}{\partial x} \\
\ddot{y}=-\frac{\partial U}{\partial y} .
\end{array}\right.
$$

From the energy relation $h=\frac{1}{2}\left(\dot{x}^{2}+\dot{y}^{2}\right)+U(x, y)$ we have that

$$
\dot{x}^{2}=\frac{2(h-U)}{1+\left(\frac{d y}{d x}\right)^{2}} .
$$

By the chain's rule

$$
\dot{y}=\frac{d y}{d x} \dot{x} \quad \text { and } \quad \ddot{y}=\frac{d^{2} y}{d x^{2}} \dot{x}^{2}+\frac{d y}{d x} \ddot{x}
$$

If we substitute the equations (5) and (6) in (4), we obtain

$$
-\frac{\partial U}{\partial y}=\frac{d^{2} y}{d x^{2}} \cdot \frac{2(h-U)}{1+\left(\frac{d y}{d x}\right)^{2}}+\frac{d y}{d x}\left(-\frac{\partial U}{\partial x}\right),
$$


or equivalently

$$
\frac{d^{2} y}{d x^{2}}=\frac{\left[\frac{\partial U}{\partial x} \cdot \frac{d y}{d x}-\frac{\partial U}{\partial y}\right] \cdot\left[1+\left(\frac{d y}{d x}\right)^{2}\right]}{2(h-U)} .
$$

For $x \gg 0$ we consider the transformation $M_{h} \rightarrow \Omega \times \mathbb{R} P^{1}$ given by $(x, y) \rightarrow(1 / x, y / x)=(z, \omega)$ where $\Omega \subset \mathbb{R}$ is a neighborhood of zero and $\mathbb{R} P^{1}$ is the one-dimensional real projective line. We have noted in $[\mathbf{L R 2}]$ that such transformation carries straight lines with the form $y=\alpha x+\beta$ into straight lines with the form $\omega=\beta z+\alpha$ in $\Omega \times \mathbb{R} P^{1}$, that is, it carries straight lines in $M_{h}$ into straight lines in $\Omega \times \mathbb{R} P^{1}$ exchanging slopes with ordinates at the origin. Under such transformation, equation (7) in the variables $(z, \omega)$ is written as

$$
z^{2} \omega^{\prime \prime}=\frac{-\left[\left(z \frac{\partial U}{\partial z}+\omega \frac{\partial U}{\partial \omega}\right)\left(\omega-z \omega^{\prime}\right)+\frac{\partial U}{\partial \omega}\right]\left[1+\left(\omega-z \omega^{\prime}\right)^{2}\right]}{2(h-U)},
$$

where' ${ }^{\prime}$ is the derivative with respect to $z$.

From the above reasoning, searching solutions $y=y(x)$ of (7) being asymptotic to a straight line of the form $y=\alpha x+\beta$ as $x \rightarrow \infty$, is equivalent to search solutions $\omega=\omega(z)$ of $(8)$ crossing the axis $\{0\} \times \mathbb{R} P^{1}$ at the point $(0, \alpha)$ with a slope $\beta$. In this way, we look for analytic solutions of the second order differential equation (8), for the initial conditions $z=0, \omega=\alpha$ and $\omega^{\prime}=\frac{d \omega}{d z}(0)=\beta$.

First we observe that (8) is a differential equation of the form

$$
z^{2} \omega^{\prime \prime}=f\left(z \omega^{\prime}, \omega, z\right)
$$

where the right hand side is an analytic function in its arguments.

In $[\mathbf{F}]$ is proven that such differential equation with singularity in $z=0$, has analytic solutions for the initial conditions $z=0, \omega=\alpha$ and $\omega^{\prime}=\beta$, if and only if the following conditions are satisfied:

a) $\alpha \in \mathbb{R} P^{1}$ is such that $f(0, \alpha, 0)=0$ and the parameter $\beta$ must satisfy $\left(f_{100}+f_{010}\right) \beta=-f_{001}$, where

$$
f_{100}=\frac{\partial f}{\partial\left(z \omega^{\prime}\right)}(0, \alpha, 0), f_{010}=\frac{\partial f}{\partial \omega}(0, \alpha, 0) \quad \text { and } \quad f_{001}=\frac{\partial f}{\partial z}(0, \alpha, 0),
$$

and

b) the critical quadratic

$$
\lambda^{2}-\lambda\left(f_{100}-3\right)+\left(2-2 f_{100}-f_{010}\right)=0
$$

associated to the differential equation (8) has no positive integers as roots. 
We consider now homogeneous potentials of degree -1 . This type of potentials appear usually in the problems of classical mechanics and have been studied $([\mathbf{L} \mathbf{1}],[\mathbf{L} 2],[\mathbf{L B I}])$. Particularly the problems of celestial mechanics and the Coulombian repulsives ones have this type of potential. We have the following result:

Theorem 3. Let $U=U(x, y)$ be homogeneous potential of degree -1 . Then the differential equation (8) has analytic solutions $\omega=\omega(z)$ which cross the straight line $\{0\} \times \mathbb{R} P^{1}$ for the initial conditions $z=0, \omega=\alpha$, $\omega^{\prime}=\beta$ if and only if $\alpha$ satisfies the relation $\alpha \tilde{U}(\alpha)+\left(1+\alpha^{2}\right) \frac{d \tilde{U}(\alpha)}{d \omega}=0$ and $\beta$ is arbitrary. Here $\tilde{U}(\omega)=U(1, \omega)$ is an analytic function depending only on $\omega$.

Proof: Let the change of coordinates be $(x, y) \rightarrow(1 / x, y / x)=(z, \omega)$; then from the homogeneity of $U$, we have

$$
U=U(x, y)=U\left(\frac{1}{z}, \frac{\omega}{z}\right)=z U(1, \omega)=z \tilde{U}(\omega),
$$

where $\tilde{U}(\omega)=U(1, \omega)$ is only a function of $\omega$.

Therefore

$$
\frac{\partial U}{\partial z}=\tilde{U}(\omega) \quad \text { and } \quad \frac{\partial U}{\partial \omega}=z \frac{d \tilde{U}}{d \omega}
$$

which implies that

$$
z \frac{\partial U}{\partial z}+\omega \frac{\partial U}{\partial \omega}=z\left[\tilde{U}(\omega)+\omega \frac{d \tilde{U}}{d \omega}\right]
$$

In this way equation (8) is written

$$
\begin{aligned}
z^{2} \omega^{\prime \prime} & =z \cdot \frac{-\left[\left(\tilde{U}(\omega)+\omega \frac{d \tilde{U}}{d \omega}\right)\left(\omega-z \omega^{\prime}\right)+\frac{d \tilde{U}}{d \omega}\right]\left[1+\left(\omega-z \omega^{\prime}\right)^{2}\right]}{2(h-z \tilde{U})} \\
& =f\left(z \omega^{\prime}, \omega, z\right) .
\end{aligned}
$$

A straightforward calculation shows that

$$
f(0, \alpha, 0) \equiv 0, \quad f_{100} \equiv 0, \quad f_{010} \equiv 0,
$$

and

$$
f_{001}=\frac{-\left[\left(\tilde{U}(\alpha)+\alpha \frac{d \tilde{U}(\alpha)}{d \omega}\right) \alpha+\frac{d \tilde{U}(\alpha)}{d \omega}\right]\left[1+\alpha^{2}\right]}{2 h}
$$


Since we have to satisfy $\beta$ arbitrary and satisfies $\left(f_{100}+f_{010}\right) \beta=$ $-f_{001}$, it is necessary and sufficient that $f_{001} \equiv 0$, which is equivalent to $\alpha \tilde{U}(\alpha)+\left(1+\alpha^{2}\right) \frac{d \tilde{U}(\alpha)}{d \omega}=0$ and $\beta$ arbitrary.

It follows that for all $\omega \in \mathbb{R} P^{1}, f(0, \omega, 0) \equiv 0, f_{100} \equiv 0$ y $f_{010} \equiv 0$, which implies that the critical quadratic associated to $\left(8^{\prime}\right)$ is $\lambda^{2}+3 \lambda+2=$ 0 , whose roots are not positive integers.

Hence, we have analytic solutions only when the initial conditions $z=0, \omega=\alpha$ satisfy the above relation and $\omega^{\prime}=\beta$ is arbitrary.

A solution of (4) is called homothetic if when it is projected in the configuration space, it has the form $(x(t), y(t))=\lambda(t)\left(x_{0}, y_{0}\right)$ for some fixed vector $\left(x_{0}, y_{0}\right) \in S^{1}$ and $\lambda(t)$ a scalar function. The vector $\left(x_{0}, y_{0}\right)$ is called a central configuration of the mechanical system.

Lemma 4. Let $\alpha$ be a direction in the configuration space for a classical mechanical system with homogeneous potential of degree -1 . Then $\alpha$ does satisfy the relation $\alpha \tilde{U}(\alpha)+\left(1+\alpha^{2}\right) \frac{d \tilde{U}(\alpha)}{d \omega}=0$ in Theorem 3 if and only if it is a central configuration of the problem.

Proof: In $[\mathbf{L B I}]$ it is shown that if the energy function in the variables $(x, y, \dot{x}, \dot{y})$ is written $H=\frac{1}{2}\left(\dot{x}^{2}+\dot{y}^{2}\right)+U(x, y)$ with homogeneous potential $U$ of degree $-1, Q_{0}=\left(x_{0}, y_{0}\right) \in S^{1}$ is a central configuration if it generates a homothetic solution and satisfies that

$$
\operatorname{grad} U\left(Q_{0}\right)=-U\left(Q_{0}\right) \cdot Q_{0} \text {. }
$$

In this way if $Q_{0}=\left(x_{0}, y_{0}\right)$ is a central configuration then we can write it in an equivalent form

$$
\left(\frac{\partial U}{\partial x}\left(Q_{0}\right), \frac{\partial U}{\partial y}\left(Q_{0}\right)\right)=-U\left(x_{0}, y_{0}\right) \cdot\left(x_{0}, y_{0}\right) .
$$

In coordinates $z, \omega$, this can be written as

$$
z^{2}\left(-\left(\tilde{U}+\omega \frac{d \tilde{U}}{d \omega}\right), \frac{d \tilde{U}}{d \omega}\right)_{Q_{0}}=\tilde{U}(\omega) \cdot(1, \omega)_{Q_{0}}
$$

where $Q_{0}=\left(x_{0}, y_{0}\right)=\left(\frac{1}{z_{0}}, \frac{\omega_{0}}{z_{0}}\right)=\frac{1}{z_{0}}\left(1, \omega_{0}\right)=\frac{1}{z_{0}}(1, \alpha)$, because $\omega_{0}=\alpha$.

The last equality is true if and only if the vectors $\left(-\left(\tilde{U}+\omega \frac{d \tilde{U}}{d \omega}\right), \frac{d \tilde{U}}{d \omega}\right)$ and $(1, \omega)$ are parallel, or equivalently

$$
\left.\operatorname{det}\left[\begin{array}{cc}
-\left(\tilde{U}+\omega \frac{d \tilde{U}}{d \omega}\right) & \frac{d \tilde{U}}{d \omega} \\
1 & \omega
\end{array}\right]\right|_{\omega=\alpha}=0
$$

which is the same as $\alpha \tilde{U}(\alpha)+\alpha^{2} \frac{d \tilde{U}(\alpha)}{d \omega}+\frac{d \tilde{U}(\alpha)}{d \omega}=0$. 
Remark. The case where the degree of homogeneity is $-k$ with an integer $k>1$ can be treated in a similar way; but in this case we have that equation $\left(8^{\prime}\right)$ becomes a non singular second order differential equation as we can verify. The results hold from the classical Fuch's Theorem on the existence of solutions for the same given initial conditions. For this reason, the case of homogeneity -1 in the potential is the most interesting when we use the methods developed here.

When the value of $\alpha$ corresponds to a central configuration we can write the solution of the differential equation (8) for the initial conditions $z=0, \omega=\alpha, \omega^{\prime}=\beta$ in the form $\omega(z)=\alpha+\beta z+a_{2} z^{2}+a_{3} z^{2}+a_{3} z^{3}+\cdots$.

When this solution is carried to the Hill's region via the inverse transformation $\Omega \times \mathbb{R} P^{1} \rightarrow M_{h}$ given by $(z, \omega) \rightarrow\left(\frac{1}{z}, \frac{\omega}{z}\right)=(x, y)$, it takes the form $y=\beta+\alpha x+\frac{a_{2}}{x}+\frac{a_{3}}{x^{2}}+\cdots$. This is a solution of (7) asymptotical to the straight line $y=\beta+\alpha x$ as $x \rightarrow \infty$. We say in this case that $\alpha$ as slope is a central direction.

We observe that in this case, the "ordinate at the origin" $\beta$ is arbitrary. If we return to the equations with the time as independent variable, we note that the parameter $\beta$ and the time $t$ must generate a two-dimensional manifold. From these considerations, we obtain the following result.

Corollary 3 (Escapes in direction of central configurations). Let $M_{h}$ be an unbounded Hill's region for the energy level $h>0$ of the problem (4). Then, for a central direction $\alpha$ of the configuration space there exists a two dimensional submanifold formed by solutions in phase space, which when projected in the Hill's region $M_{h}$, become parallel to the straight line $y=\alpha x$ as $t \rightarrow \infty$.

Now, for studying escapes in any other given direction, we proced as we did in [LR2] using the coordinates change $z=\tau^{\rho}$. Then if we substitute in $\left(8^{\prime}\right)$, obtain the new second order differential equation in the variables $\tau, \omega$ :

$$
\begin{aligned}
\left(8^{\prime \prime}\right) \tau \ddot{\omega}=\frac{-\rho^{2} \tau^{\rho-1}\left[\left(\tilde{U}(\omega)+\omega \frac{d \tilde{U}}{d \omega}\right)\left(\omega-\frac{\tau}{\rho} \dot{\omega}\right)+\frac{d \tilde{U}}{d \omega}\right]\left[1+\left(\omega-\frac{\tau}{\rho} \dot{\omega}\right)^{2}\right]}{2\left(h-\tau^{\rho} \tilde{U}\right)} & +(\rho-1) \dot{\omega},
\end{aligned}
$$

which can be treated in a similar way for finding solutions crossing the axis $\{0\} \times \mathbb{R} P^{1}$. An elaborated analysis shows that $\left(8^{\prime \prime}\right)$ has analytic solutions in the variables $\tau, \tau^{\rho-1}$ for the initial conditions $\tau=0, \omega=\alpha$ and $\dot{\omega}=\beta$ if and only if $\omega(0)=\alpha$ is arbitrary and $\dot{\omega}=\beta=0$ (see $[\mathbf{F}]$ ). 
In fact for the initial conditions obtained in this way there exist infinitely many solutions of $\left(8^{\prime \prime}\right)$ whose general form is

$$
\omega(\tau)=\alpha+a_{\rho-1} \tau^{\rho-1}+\sum_{m, n \geq 2}^{\infty} a_{m n} \tau^{m(\rho-1)} \tau^{n},
$$

where $a_{\rho-1}$ is an arbitrary constant and the coefficients $a_{m n}$ in the series do depend of $\alpha$ and $a_{\rho-1}$. We remark that we have chosen $\rho$ such that $2<\rho<3$.

When we carry these solutions into Hill's region via the inverse transformation $(x, y) \rightarrow\left(\frac{1}{x^{1 / \rho}}, y / x\right)$, they take the form

$$
y=\alpha x+a_{\rho-1} x^{1 / \rho}+\sum_{m, n \geq 2}^{\infty} a_{m n} x^{m(1 / \rho-1)} x^{1-n / \rho}
$$

having a limiting slope at infinity equal to $\alpha$, but they are not all asymptotical to a straight line of the form $y=\alpha x+\beta$ as $x \rightarrow \infty$.

When we fix the direction $\alpha$, the $a_{\rho-1}$ is an arbitrary constant of integration not depending of the initial conditions $\omega(0)=\alpha, \beta=0$ of the diferential equation $(7)$ (see $[\mathbf{F}]$ ). Then $a_{\rho-1}$ generate a one-parameter family of solutions on the Hill's region which escape in the direction $\alpha$. These results rederive constructively the one obtained by Lacomba in [L2] on the existence of a two-dimensional submanifold formed by orbits which escape in each direction $\alpha$, and all the projected solutions escaping in this direction can be written in this way:

Theorem 4. Let $U$ be a homogeneous potential of degree -1, and let us fix the energy $h>0$. Then, for a given direction $\alpha$ of escape in the configuration space, there exists a two dimensional submanifold in the phase space formed by solutions of (4), which when projected into the Hill's region become parallel or asymptotic to the straight line $y=\alpha x$ as $t \rightarrow \infty$.

We note that in this case we impose the unboundedness condition on the Hill's region and no topological condition; the direction on which there are parallel escapes are constrained to the possible escapes in the Hill's region: the blowing up used for studying those escapes must be able to detect such constraints in particular problems. We refine the above result in the following section, by imposing suitable conditions on the potential $U$. In that case, escape in any direction will always be in such a way that the geodesics become asymptotically parallel among themselves. In fact, the above two dimensional submanifold projects nicely into a foliation of $\left(\operatorname{int} M_{h}\right)$. We give examples of this in Section 5 . 


\section{Compactification of the Hill's region}

From now on we assume that the potential $U(x, y)$ satisfies any one of the three conditions in Corollary 1 , so that the conformal metric $g_{h}$ has negative curvature for a fixed energy level. We also assume that $U$ is homogeneous of degree -1 , so that escape geodesics have limiting directions, as shown in Section 3. For any negative degree of homogeneity of the potential any escape solution has an asymptotic direction at infinity. This can be checked as in $[\mathbf{L 2}]$, so the results below can be extended to this general case.

We recall that the zero velocity curve is defined by $\Gamma=\{(x, y) \in$ $\left.M_{h} \mid U(x, y)=h\right\}$ and is the boundary of the Hill's region. We recall also that classical mechanical systems are reversible. This means that if $\gamma(t)$ is the projection on the Hill's region of a solution curve in the phase space which intersects the curve $\Gamma$ at time $t=0$, then for any time $t$ we have that $\gamma(t)=\gamma(-t)$ which can be verified in (4) by a simple substitution. Hence, we can contruct a local one-dimensional foliation of a neighborhood of the zero velocity curve $\Gamma$ contained in $M_{h}$, formed by the projections of the local solutions in phase space which touch it. This is because of the uniqueness of solutions with respect to initial conditions on $\Gamma$. In fact, the above one dimensional foliation of geodesics touching $\Gamma$ is global. This is proved in Theorem 5 .

We study now the flow of problem (4) as the geodesic flow in $\operatorname{int}\left(M_{h}\right)$ associated to the metric $g_{h}$, parametrized by arc length. We begin with the following result.

Theorem 5. For any point $P \in \operatorname{int}\left(M_{h}\right)$ there exists one and only one geodesic curve through $P$ whose projection intersects the zero velocity curve $\Gamma$.

Proof: (Existence): Let $P$ be an arbitrary point in $\operatorname{int}\left(M_{h}\right)$. If there is no such a curve, then all curves through $P$ escape, because there are neither non trivial critical points nor periodic orbits (Gauss-Bonnet Theorem for manifolds with negative curvature). By Lemma 1 all of them are defined for all the values of the parameter $t \in \mathbb{R}$, which implies that the system is complete in $P$. Therefore, $\operatorname{int}\left(M_{h}\right)$ is complete itself because it is connected and simply connected. This is not posible because there are curves begining in the zero velocity curve and from Lemma 2 the points on that curve have finite distance from $\Gamma$. Then there exists a geodesic which we denote by $\gamma_{P}$ converging to $\Gamma$.

From Gauss-Bonnet Theorem again, $\gamma_{P}$ intersects any geodesic of the local foliation of a neigborhood of $\Gamma$ in at most one point, and therefore $\gamma_{P}$ does not acumulate in $\Gamma$, and is one of the leaves of the local foliation. 
(Uniqueness): If there are two geodesics through $P$ converging to the zero velocity curve in finite time, it follows from Gauss-Bonnet that any geodesic between them converges also to the zero velocity curve. Let us refer to such set of geodesics as a pencil with vertex $P$.

We claim that the above mentioned pencil is bounded. If it is not the case, then we choose a point $Q$ outside the pencil, and consider a geodesic $\gamma_{Q}$ through $Q$ converging to the zero velocity curve and intersecting $\Gamma$ at the point $Q_{0}$. This point is contained in the unbounded pencil with vertex $P$ and therefore there exists a geodesic $\gamma_{P}$ through $P$ converging to $Q_{0}$. This is not posible because of the uniqueness of the physical solution through $Q_{0} \in \Gamma$ with zero initial velocity. Since the above reasoning is symmetrical, the aforementioned pencil is bounded.

Let $\gamma_{2}$ be the lower geodesic for the pencil, and $\gamma_{1}$ an arbitrary geodesic in the pencil. If the corresponding points for these geodesics in $\Gamma$ are $Q_{2}$ and $Q_{1}$ respectively, then all the intermediate geodesics from $P$ converge to $\Gamma$ between $Q_{1}$ and $Q_{2}$. Now, by using continuity respect to initial conditions, we can choose a point $Y \in \Gamma$ below $Q_{2}$ (outside the pencil) such that the unique geodesic going to $Y$ intersects the other half part of the pencil (with vertex in $P$ ) at the point $R \neq P$. For such a point $R$ passes a geodesic which also passes through $P$ and converges to the zero velocity curve at the point $R_{0}$.

We consider now the pencil with vertices $P, Q_{1}, Q_{2}$, then, any geodesic reaching between $Q_{1}$ and $Q_{2}$ passes through $P$. Similarly, for the pencil with vertices $R, R_{0}, Y$, any geodesic reaching between $R_{0}$ and $Y$ passes through $R$. Therefore, for any geodesic arising between $R_{0}$ and $Q_{2}$ we have that simultaneously passes through $P$ and passes through $R$. This is not possible because of the uniqueness of the solutions reaching the zero velocity curve. Therefore, there is only one geodesic passsing through $P$ and converging to $\Gamma$ in finite time.

Definition. Let $P \in \operatorname{int}\left(M_{h}\right)$ be an arbitrary point. The polar neighborhood $B_{\rho}(P)$ of radius $\rho$ of $P$, is a neighborhood contained in $\operatorname{int}\left(M_{h}\right)$ such that all the points inside can be joined by a minimizing geodesic of length smaller than $\rho$.

We obtain the following lemma on distances between arbitrary points in the interior of Hill's region, via points in the boundary of polar neighborhoods. We define the distance from $P \in \operatorname{int}\left(M_{h}\right)$ to $\Gamma$, denoted $d(P, \Gamma)$, as the distance from $P$ to $P_{0}$, where $P_{0}$ is the point of intersection with $\Gamma$ of the unique geodesic through $P$ given by Theorem 5 . 
Lemma 5. For any pair of points $P, Q \in \operatorname{int}\left(M_{h}\right)$ and any polar neighborhood of $P$ with radious $\rho<d(P, \Gamma)$, there exists one point $R$ in the circle $S_{\rho}(P)=\partial\left(B_{\rho}(P)\right)$ such that

$$
d(P, R)+d(R, Q)=d(P, Q) .
$$

Proof: Since the circle $S_{\rho}$ is compact, then there exists a point $R^{*} \in S_{\rho}$ such that

$$
d\left(P, R^{*}\right)+d\left(R^{*}, Q\right)
$$

is minimal.

Let us suppose that

$$
d\left(P, R^{*}\right)+d\left(R^{*}, Q\right)=d(P, Q)+\eta
$$

for some number $\eta>0$.

For $\epsilon>0$ there exists a curve $\gamma$ such that

$$
d(P, Q)+\epsilon=\operatorname{long}(\gamma)
$$

If we take $\epsilon=\frac{\eta}{2}$, then

$$
d\left(P, R^{*}\right)+d\left(R^{*}, Q\right)=\eta+\operatorname{long}(\gamma)-\epsilon=\frac{\eta}{2}+\operatorname{long}(\gamma)
$$

which implies that

$$
d\left(P, R^{*}\right)+d\left(R^{*}, Q\right)>\operatorname{long}(\gamma)
$$

contradicting the fact that at $R^{*}$ we obtain the minimum over all the curves joining the points $Q$ and $P$. This proves the lemma if we put $R=R^{*}$.

The following result is a generalization of the Lemma 2.1.2 in Klingenberg $[\mathbf{K l}]$ for non-complete connected, simply connected surfaces with negative curvature.

Proposition 3. For any pair of points $P$ and $Q$ in the interior of the Hill's region there exists a minimizing geodesic joining them.

Proof: Let us consider again the unique geodesic $\gamma_{0}$ through $P$ converging to the zero velocity curve at the point $P_{0}$, and let $\tilde{\rho}=d\left(P, P_{0}\right)<$ $\infty$ be the radius of the maximal polar neighborhood. 
If $Q \in \gamma_{0}$ or $Q \in B_{\tilde{\rho}}$, then there is nothing to prove, because from Lemma 2.1.2 in $[\mathbf{K l}]$ the exponential map at $P$ is defined in $B_{\tilde{\rho}}(0) \subset$ $T_{P}\left(M_{h}\right)$, and the proposition holds on $B_{\tilde{\rho}}(P)$.

So, we suppose $Q$ is neither in $\gamma_{0}$ nor in $B_{\rho}$. From Lemma 5 given $0<\delta<\tilde{\rho}$, there exists $R \in S_{\delta}(P)$ such that

$$
d(P, R)+d(R, Q)=d(P, Q) .
$$

Let us denote by

$$
\left\{R_{\delta}, R_{\delta}^{\prime}\right\}=\gamma_{0} \cap S_{\delta}(P)
$$

the points of intersection between $\gamma_{0}$ and $S_{\delta}$.

We claim that $R \neq R_{\delta}$ and $R \neq R_{\delta}^{\prime}$ for every $0<\delta<\tilde{\rho}$. If this were not true, consider the set

$$
\Delta=\left\{\delta \mid 0 \leq \delta<\tilde{\rho}, R_{\delta} \in \gamma_{0}, \quad \text { and } \quad d\left(P, R_{\delta}\right)+d\left(R_{\delta}, Q\right)=d(P, Q)\right\} .
$$

It is clear because of the continuity of distance that $\Delta$ is a closed non empty subset of $\mathbb{R}$. Let $\delta^{*}=\sup \Delta$.

For given $\epsilon>0$, let $\gamma_{R_{\delta^{*}}, Q}$ be a curve such that

$$
\epsilon+d\left(R_{\delta^{*}}, Q\right)=\operatorname{long}\left(\gamma_{R_{\delta^{*}}, Q}\right)
$$

and consider the point

$$
R^{*}=\gamma_{R_{\delta^{*}}, Q} \cap S_{\rho_{1}}
$$

for some $\rho_{1}$, with $\delta^{*}<\rho_{1}<\tilde{\rho}$.

From the definition of $\delta^{*}$, is clear that $R^{*}$ is not in $\gamma_{0}$. Then, we consider the minimizing geodesic joining $P$ and $R^{*}$. Because the distances in $B_{\tilde{\rho}}(P)$ are attained at the minimizing geodesics, then the curve joining $P$ with $R_{\delta^{*}}$ and $R_{\delta^{*}}$ with $R^{*}$ can not minimize the distance between $P$ and $R^{*}$ unless it is itself a geodesic. This contradicts the uniqueness of the geodesics inside $B_{\tilde{\rho}}$, because $R^{*}$ does not belong to the geodesic $\gamma_{0}$. Therefore $R \neq R_{\delta}$ for all $0<\delta<\tilde{\rho}$. Similarly $R \neq R_{\delta}^{\prime}$.

To complete the proof, we use the fact that we can extend to all the real numbers any geodesic through $P$ different from $\gamma_{0}$. The same analysis in the proof of Lemma 2.1.2 in [Kl] follows for this case, and we omit the details.

Because of the Lemmas 1 and 2, given any geodesic $\gamma$, its maximal domain of definition has to be one of the following three types of intervals

a) $J=\left(-\infty, a_{0}\right)$ (it reaches $\Gamma$ in finite time as time increases).

b) i) $J=\left(a_{0},+\infty\right)$ (it escapes to $\infty$, arising from $\Gamma$ ).

ii) $J=\mathbb{R}$ (it escapes if $t \rightarrow \pm \infty$ ). 
We say that a geodesic $\gamma$ with domain of definition as in a) is positively singular. We say that it is negatively singular if its domain falls in the case b) i). Otherwise if $J=\mathbb{R}$, we say that it is regular.

Definition. Let $\gamma_{1}: J_{1} \rightarrow M_{h}, \gamma_{2}: J_{2} \rightarrow M_{h}$ be two geodesics in the interior of $M_{h}$. We say the geodesics are positively asymptotic if

a) Both converge to points in $\Gamma$ in finite time, as the time increases, or

b) Both escape to infinite as $t \rightarrow \infty$, and they have the same limiting direction at $\infty$.

Similarly we define when two geodesics are negatively asymptotic. The following lemma is an immediate consequence of the last definition.

Lemma 6. The positively (negatively) asymptotic relation between geodesics is an equivalence relation.

In the "direction" of $\Gamma$ there exists a foliation of the geodesic flow in a neighborhood of $\Gamma$ formed by geodesics intersecting the zero velocity curve. Even more, from Theorem 5 such a foliation is global.

In this way, when $U$ is a homogeneous potential of degree -1 , we have that for every escape direction $z=\arctan (\alpha)$ in the configuration space there exists an infinity of asymptotic geodesics which are determined by their limiting direction $z$ at infinity. We prove below that those geodesics foliate $\operatorname{int}\left(M_{h}\right)$ for any given direction.

We denote by $z_{0}$ the direction corresponding to $\Gamma$. That is, if a geodesic $\gamma \subset M_{h}$ reaches $\Gamma$, then it has as limiting direction $z_{0}$.

Theorem 6. Given any direction $z=\arctan (\alpha)$ in the Hill's region and any point $P \in \operatorname{int}\left(M_{h}\right)$ there exists a unique geodesic $\gamma_{P}$ through $P$ and having positively asymptotic direction $z$. Analogously for the negatively asymptotic case.

Proof: Let $P \in \operatorname{int}\left(M_{h}\right)$ be an arbitrary point and let $z$ be any given direction. If $z=z_{0}$, then Theorem 5 proves the result. The other curves through $P$ escape to $\infty$. If $z \neq z_{0}$, then from Theorem 4 there exists a two dimensional submanifold in phase space formed by geodesics $\gamma(t)$ such that all these satisfy $\gamma(t) \rightarrow \infty$ when $t \rightarrow \infty$ and have as limiting direction $z$. Let $\gamma_{z}(t)$ be any of these geodesics such that it does not arise from $\Gamma$ (by Theorem 5 there is at most one coming from $\Gamma$ ) and suppose that $P$ is not in $\gamma_{z}$. Then $\gamma_{z}$ is defined for all $t \in \mathbb{R}$. 
If we consider the distance function $d(t)=d\left(P, \gamma_{z}(t)\right)$, such a function is convex $\left(d^{\prime \prime}(t) \geq 0\right)$, never vanishes, and it is of class $C^{1}$ (see Proposition 3.8.1 in $[\mathbf{K l}])$. Therefore, $d$ attains its minimum value at some $t=t_{0}$. From Lemma 3.8.2 in $[\mathbf{K l}]$, the geodesic joining the points $P$ and $\gamma_{z}\left(t_{0}\right)$ is orthogonal to $\gamma_{z}$ at the point $\gamma_{z}\left(t_{0}\right)$. Let $\left\{n: n \geq t_{0}\right\}$ be a sequence of positive integers going to infinity, and consider the infinite sequence of points $\left\{\gamma_{z}(n)\right\} \subset \gamma_{z}(\mathbb{R})$. For any integer $n$ in the sequence there is a unique geodesic $\gamma_{n}(t)$ joining the points $P$ and $\gamma_{z}(n)$. If $\gamma_{n}(0)=P$ and we put $\dot{\gamma}_{z}(0)=v_{n}$, then the sequence of tangent vectors $\left\{v_{n}\right\} \subset S^{1} \subset T_{P} M_{h}$ has an accumulation point namely $v$. As in Lemma 3.8.5 of Klingenberg [Kl] , if we define the geodesic $\gamma_{P}(t)$ as the one satisfying the initial conditions $\gamma_{P}(0)=P$ and $\dot{\gamma}_{P}(0)=v$, this is the desired geodesic. Uniqueness follows as in Lemma 3.8.5 in $[\mathbf{K l}]$ and we omit the details.

Morever, also in the same Lemma 3.8.5 in $[\mathbf{K l}]$, it is proven that the distance between the points $\gamma_{P}(t)$ and $\gamma_{z}(t)$ is bounded for $t \geq t_{0}$. That is, there exists a positive number $K=d\left(P, \gamma_{P}\left(t_{0}\right)\right)$ such that if $t \geq t_{0}$ then $d\left(\gamma_{P}(t), \gamma_{z}(t)\right) \leq 3 K-t_{0}$. From this and Theorem 4 we obtain the following result which generalizes the one on asymptotic parallel escapes in the direction of the central configurations given in [LR2] for isosceles 3-body problems, and for positive energy.

Corollary 4. Any pair of geodesics $\gamma_{1}$ and $\gamma_{2}$ escaping in the same direction at infinity are asymptotically parallel. That is, for some big enough $t_{0}$ there exists a positive number $K$ such that if $t \geq t_{0}$ then

$$
d\left(\gamma_{1}(t), \gamma_{2}(t)\right) \leq K .
$$

The set of asymptotic geodesics at infinity in a given direction $z=$ $\arctan (\alpha)$ will be called the geodesic pencil in that direction. If there is no confusion we denote the pencil by the same symbol $z$ corresponding to its direction. For each pencil $z$ at infinity, we denote by $z^{+}$the class of geodesics reaching $z$ at $+\infty$, and by $z^{-}$the class arising $z$ at $-\infty$. As foliations, $z^{+}$and $z^{-}$are the same object but the corresponding curves of each class have opposite directions.

The singular geodesic pencil corresponding to the direction $z_{0}$ is the set of geodesics reaching $\Gamma$. The direction $z_{0}$ is singular in the sense that geodesics getting there, arrive in finite time and in the physical system they retrace themselves (because of the reversibility), while geodesics arrive at any other direction asymptotically in infinite time. Similarly to the case at $\infty$, we denote by $z_{0}^{+}$the class of geodesics arriving to $\Gamma$ as 
the time increases, and by $z_{0}^{-}$to the class of geodesics arising from $\Gamma$ as the time increases.

We consider now the case where the boundary $\Gamma$ is a connected curve with two asymptotic straight lines $L_{\alpha}, L_{\beta}$ having limiting directions $z_{\alpha}$ and $z_{\beta}$ at $\infty$ respectively.

In such case, the directions $z$ at $\infty$ are bounded by $z_{\alpha}<z<z_{\beta}$.

Definition (Boundary of $M_{h}$ ). The geodesic pencils are called the points at infinity of $M_{h}$ and the set formed by all of them is denoted by $M_{h}(\infty)$. This set of points at infinity is called the ideal boundary or absolute of $M_{h}$.

The closure of $M_{h}(\infty)$ will be the set

$$
\overline{M_{h}}(\infty)=M_{h}(\infty) \cup\left\{z_{0}\right\},
$$

and the closure of $M_{h}$ will be $\overline{M_{h}}=\overline{M_{h}}(\infty) \cup \operatorname{int}\left(M_{h}\right)$.

If $\gamma: J \rightarrow M_{h}$ is a regular or negatively singular geodesic then $\gamma(+\infty)$ will denote the corresponding class of geodesics which contains it, and it can be identified with the limiting direction when the parameter tends to $+\infty$. In a similar way if $\gamma$ is regular or positively singular, $\gamma(-\infty)$ will denote the corresponding class of geodesics which contains it as the parameter tends to $-\infty$.

The following result is an immediate consequence of Theorem 6 .

Corollary 5. If $P \in \operatorname{int}\left(M_{h}\right)$ and $z \in M_{h}(\infty)$, then there exists a unique geodesic $\gamma \subset M_{h}$ defined for any big enough positive time, such that $\gamma(0)=P$ and $\gamma(+\infty)=z^{+}$. Similarly for the case $\gamma(0)=P$ and $\gamma(-\infty)=z^{-}$.

The following result is a direct consequence of Theorem 5.

Corollary 6. If $P \in \operatorname{int}\left(M_{h}\right)$, then there exists a unique geodesic $\gamma \subset M_{h}$ defined in an upper unbounded interval, such that $\gamma(0)=P$ and $\gamma \in z_{0}^{+}$. Similarly for the case $\gamma(0)=P$ and $\gamma \in z_{0}^{-}$.

We shall denote by $J=(a, b)$ any of the intervals defining the type of aforementioned geodesics of the problem. We understand that $a<b$ could be any of the extremes of such type of intervals. For example, $a=a_{0}$ and $b=\infty$ for the case b) i). From Theorems 5 and 6 , every direction $z \in \overline{M_{h}}(\infty)$ defines two oriented foliations $z^{-}$and $z^{+}$of $\operatorname{int}\left(M_{h}\right)$, given by the corresponding classes, whose geodesics forming each one are the same ones but in opposite directions and are defined on suitable domains. Because every pencil foliates totally $\operatorname{int}\left(M_{h}\right)$, then we have obtained the following theorem. 
Theorem 7. Given two points $z_{1}, z_{2}$ in $\overline{M_{h}}(\infty)$, there exists a unique geodesic $\gamma(t)$ such that $\gamma \subset z_{1}^{-}$and $\gamma \subset z_{2}^{+}$.

Therefore, given two directions in the configuration space there exists a unique solution of (4) whose limiting directions are the given ones. This is summarized as

Corollary 7. Any geodesic $\gamma \subset \overline{M_{h}}$ can be written in a unique way $\gamma=z_{1}^{-} \bigcap z_{2}^{+}$as the intersection of two classes of geodesics.

An equivalent way of stating the result in Corollary 7 is by claiming that any geodesic $\gamma$ lies in only two distinct geodesic pencils: a stable one $z_{2}^{+}$and an unstable one $z_{1}^{-}$. Figure 1 illustrates this.

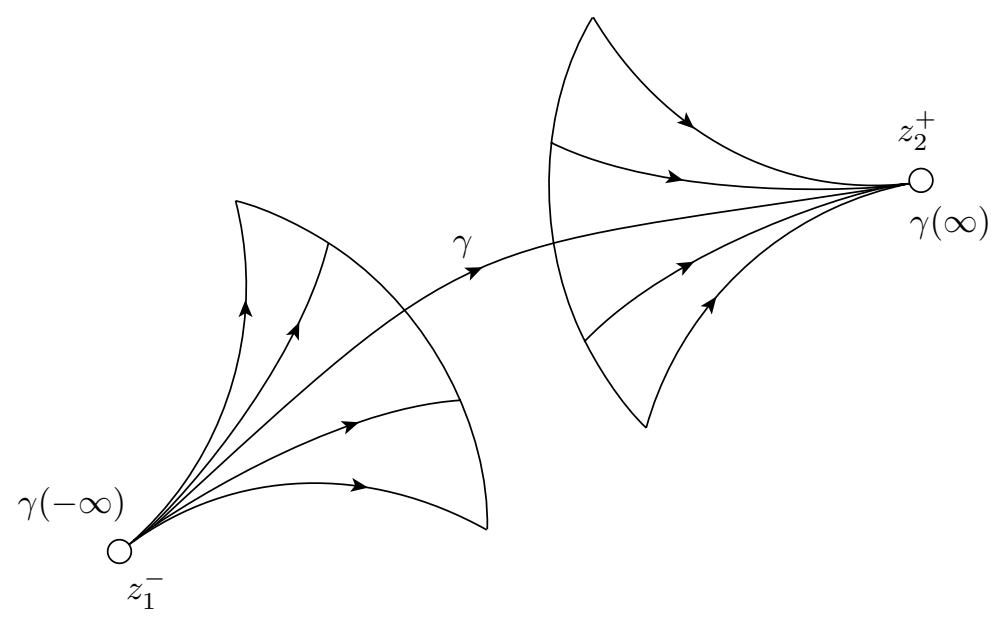

Figure 1. Stable and unstable geodesic pencils in the compactification.

We will now see which is the topology of the ideal boundary $\overline{M_{h}}(\infty)$ when $M_{h}$ is a connected, simply connected region, and $\Gamma$ is the connected boundary of $M_{h}$ having limiting directions as we mentioned before. Two classical examples of repulsive problems with these properties are given in Section 5.

For $J=(a, b)$ defining the domain of a geodesic passing through $P$, we assume $0 \in J$, in such a way that $\gamma(0)=P$.

We define below a topology for $\overline{M_{h}}$. 


\section{Definition.}

i) Let $P \in \operatorname{int}\left(M_{h}\right), z_{1}, z_{2} \in \overline{M_{h}}$ be points such that $P \neq z_{1}, P \neq z_{2}$. We define the angle between $z_{1}$ and $z_{2}$ from $P$ as $\operatorname{ang}_{P}\left(z_{1}, z_{2}\right)=$ ang $\left(\dot{\gamma}_{1}(0), \dot{\gamma}_{2}(0)\right)$ where $\gamma_{i}: J_{i} \rightarrow M_{h}$ are the geodesics going from $P$ to $z_{i}$ respectively.

ii) Given $P \in \operatorname{int}\left(M_{h}\right), z \in \overline{M_{h}}(\infty)$ and $\epsilon>0$, we define the open cone of radius $\epsilon$ with vertex at $P$ as the set $C_{P}(z, \epsilon)=\left\{\omega \in \overline{M_{h}} \mid\right.$ $\omega \neq P$ and $\left.\operatorname{ang}_{P}(z, \omega)<\epsilon\right\}$.

From Gauss-Bonnet Theorem, and Theorems 5 and 6, and since $k(x, y) \leq 0$, any open cone is a sector with vertex $P$, and it is foliated by the geodesics through $P$ having limiting directions contained in the interval $(z-\epsilon, z+\epsilon) \subset \overline{M_{h}}(\infty)$. In fact, if $\omega=\gamma\left(t_{0}\right)$ for some geodesic through $P$ inside the cone and some $t_{0} \in J$, then, for any increasing time $t$ we have $\operatorname{ang}_{P}(z, \omega)=\operatorname{ang}_{P}(z, \gamma(t))$.

We endow the set $\overline{M_{h}}$ with the topology generated by the open sets in $\operatorname{int}\left(M_{h}\right)$ and the set of all the open cones $[\mathbf{G r}]$.

We observe that a sequence of points $\left\{\omega_{i}\right\} \subset \operatorname{int}\left(M_{h}\right)$ converges to $z \in \overline{M_{h}}(\infty)$ if and only if, for every fixed point $P \in \operatorname{int}\left(M_{h}\right), d\left(P, \omega_{i}\right) \rightarrow$ $d(P, z)$ and $\operatorname{ang}_{P}\left(\omega_{i}, z\right) \rightarrow 0$. Here $d(P, z)$ is defined as $\infty$ if $z \neq z_{0}$ and as $d(P, \Gamma)$ if $z=z_{0}$. An equivalent statement is that the geodesic arcs going from $P$ to $\omega_{i}$ converge to the geodesic arc from $P$ to $z$.

The following result generalizes the Theorem 2.6.6 of Klingenberg [Kl] for complete manifolds with negative curvature.

Lemma 7. Let $P \in \operatorname{int}\left(M_{h}\right)$ be an arbitrary point and let $v_{0} \in T_{P} M_{h}$ be such that the unique geodesic $\gamma_{0}$ through $P=\gamma_{0}(0)$ with initial velocity $\dot{\gamma}_{0}(0)=v_{0}$ converges to $\Gamma$ at finite time as the time increases. Then the following restriction of the exponential map, which is defined by restricting the geodesic flow to fixed point $P$

$$
\exp _{P}: T_{P} M_{h}-\left\{t v_{0} \mid t \geq 0\right\} \rightarrow M_{h}-\left\{\gamma_{0}(t) \mid t \geq 0\right\}
$$

is a diffeomorphism.

Proof: From Theorem 5 and Proposition 3 the involved geodesics are defined for any positive time, and any pair of points inside $\operatorname{int}\left(M_{h}\right)$ can be joined by a minimizing geodesic. Now the proof follows as in Theorem 2.6.6 of Klingenberg [Kl], and we omit the details.

In fact, since $v_{0} \neq 0$, we can choose $v_{0}$ such that $\left\|v_{0}\right\|=1$ since $\gamma_{0}(t)$ is a minimizing geodesic from $P$ to $\Gamma$. 
Theorem 8 (Topology of $\overline{M_{h}}(\infty)$ ). The set $\overline{M_{h}}(\infty)$ is homeomorphic to $S^{1}$.

Proof: Let $z_{\alpha}<z_{\beta}$ be the asymptotic directions at infinity of $\Gamma$. Then the set of possible directions at infinity in $\operatorname{int}\left(M_{h}\right)$ is the interval $\left[z_{\alpha}, z_{\beta}\right]$. From Theorem 6 , for each $z$ satisfying $z_{\alpha}<z<z_{\beta}$ there is a pencil in that direction foliating $\operatorname{int}\left(M_{h}\right)$. Hence, $z \in \overline{M_{h}}(\infty)$. We claim that there are no geodesics escaping in the directions $z_{\alpha}$ or $z_{\beta}$. Indeed, if there were any geodesic $\gamma_{\alpha}$ escaping in the direction $z_{\alpha}$, by taking a point $P$ lying on $\gamma_{\alpha}$ and considering the geodesic $\gamma_{0}$ through $P$ converging to $\Gamma$, we have a region bounded by $\gamma_{\alpha}$ and $\gamma_{0}$. By Gauss-Bonnet Theorem, this region is foliated by geodesics escaping to infinity in the same direction $z_{\alpha}$. We take another such geodesic $\gamma$ inside the region and consider the distance function $f(t)=d\left(\gamma_{\alpha}(t), \gamma(t)\right)$ defined for $t \geq 0$. This function is not convex because both geodesics are asymptotically parallel. This is a contradiction because the curvature is non positive (see Proposition 3.8.1 in $[\mathbf{K l}]$ ). A similar reasoning holds for $z_{\beta}$. This completes the proof of the claim.

From Theorems 5 and 6 we know that any geodesic either escapes to infinity or approaches $\Gamma$. Since the directions $z_{\alpha}$ and $z_{\beta}$ do not have any associated pencils, it is natural to identify them with $z_{0}$, which does have its associated pencil. Topologically, this identifies the end points of $\left[z_{\alpha}, z_{\beta}\right]$, getting $S^{1}$ as required.

Corollary 8. $\overline{M_{h}}$ is homeomorphic to the closed unit disk in $\mathbb{R}^{2}$.

Proof: Since $\operatorname{int}\left(M_{h}\right)$ is connected, simply connected, and its boundary $\overline{M_{h}}(\infty)$ is homeomorphic to $S^{1}$, by the Invariance of Domain Theorem $[\mathbf{A m}]$, the assertion follows.

Finally, such a disk has complete geodesics in any direction except in the direction of $z_{0}$. We call such system quasi-complete.

We summarize now all the conditions for establishing our main result.

Consider a Hamiltonian system with two degrees of freedom, with energy function of the form

$$
E=\frac{1}{2} \delta_{i j} \dot{x}^{i} \dot{x}^{j}+U(x, y)
$$

where $x^{1}=x, x^{2}=y$.

Assume that $U$ is a homogeneous potential of degree -1 without either critical points or singularities, bounded at infinity, and $\Delta U \geq 0$. 
For a fixed energy level $h>0$, the Hill's region of the system $M_{h}$ is connected and simply connected with an unbounded connected boundary curve $\Gamma$ having limiting directions at infinity (see Figure 2).

Theorem 9. The set of physical curves in the configuration space $M_{h}$ is related, as a one-dimensional foliation, to the geodesic flow of a space homeomorphic to the unit disk, associated to a conformal quasi-complete metric with negative curvature.
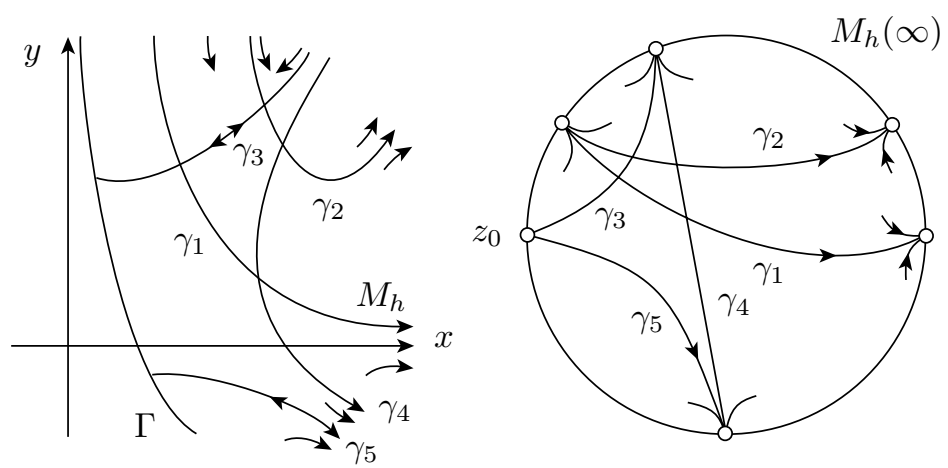

Figure 2. Hamiltonian flow and its related geodesic flow in the unit disk with negative curvature.

\section{Examples}

\subsection{The Coulombian Isosceles Problem.}

In $[\mathbf{L R 2}]$ has been shown that in Jacobi cordinates with center of mass at the origin, the equations of motion for the isosceles 3-body problem are given by (see Figure 3)

$$
\left\{\begin{array}{l}
\ddot{x}=\frac{\gamma m}{4 x^{2}}+\frac{\gamma \mu x}{\left(x^{2}+y^{2}\right)^{3 / 2}}, \\
\ddot{y}=\frac{\gamma(\mu+2 m) y}{\left(x^{2}+y^{2}\right)^{3 / 2}} .
\end{array}\right.
$$

Here we take $\gamma>0$ for the Coulombian repulsive problem and $\gamma<0$ for the Newtonian gravitational problem. The masses $\mu$ and $m$ are positive parameters.

We study the flow for the repulsive case $(\gamma>0)$ and an energy level $h>0$. 
After a linear transformation of coordinates in the configuration space $M_{h}$ given by $(x, y) \rightarrow\left(\sqrt{2 m} x, \sqrt{\frac{2 m \mu}{2 m+\mu}} y\right)$ the energy relation be-
comes

$$
h=\frac{1}{2}\left[\dot{z}^{2}+\dot{\omega}^{2}\right]+\frac{\gamma N m^{2}}{2 z}+\frac{2 \gamma M N m \mu}{\left(M^{2} z^{2}+N^{2} \omega^{2}\right)^{1 / 2}}
$$

where $z=\sqrt{2 m} x, \omega=\sqrt{\frac{2 m \mu}{2 m+\mu}} y, N=\sqrt{2 m}$ and $M=\sqrt{\frac{2 m \mu}{2 m+\mu}}$.

By relabeling the variables $z$ and $\omega$ by the old variables $x$ and $y$, we study the flow in the Hill's region

$$
M_{h}=\left\{(x, y) \mid \frac{\gamma N m^{2}}{2 x}+\frac{2 \gamma M N m \mu}{\left(M^{2} x^{2}+N^{2} y^{2}\right)^{1 / 2}} \leq h\right\}
$$

Then, the potential function in such variables is written

$$
U(x, y)=\frac{\gamma m^{2} N}{2 x}+\frac{2 \gamma \mu M N m}{\left(M^{2} x^{2}+N^{2} y^{2}\right)^{1 / 2}}
$$

A straightforward calculation in order to estimate the curvature, shows that

$$
\Delta U=\frac{\gamma m^{2} N}{x^{3}}+\frac{2 \gamma m \mu M N\left[\left(2 M^{2}-N^{2}\right) M^{2} x^{2}+\left(2 N^{2}-M^{2}\right) N^{2} y^{2}\right]}{\left(M^{2} x^{2}+N^{2} y^{2}\right)^{5 / 2}} .
$$

Therefore $\Delta U \geq 0$ if $2 M^{2}-N^{2} \geq 0$ and $2 N^{2}-M^{2} \geq 0$, and this holds if $\mu \geq 2 m$.

The Hill's region for the fixed energy level $h>0$ is a region in the plane which is connected, simply connected, while the potential $U$ is bounded at infinity and homogeneous of degree -1 . Morever, the connected boundary curve $\Gamma=\{h=U(x, y)\}$ of $\operatorname{int}\left(M_{h}\right)$ has limiting directions $z_{\alpha}=-\frac{\pi}{2}$ and $z_{\beta}=+\frac{\pi}{2}$.

In this way, by Theorem 4 and Corollary 4 , there exist asymptotic parallel escapes in every direction of the configuration space. Summarizing all this, we get

Theorem 10 (Geometry of the Repulsive Isosceles Problem). If the masses satisfy the relation $\mu \geq 2 m$, we have that the set of physical curves of this problem is related, as a one-dimensional foliation, to the geodesic flow in a space homeomorphic to the unit disk, with respect to a conformally Euclidean quasi-complete metric with negative curvature (see Figure 3). 

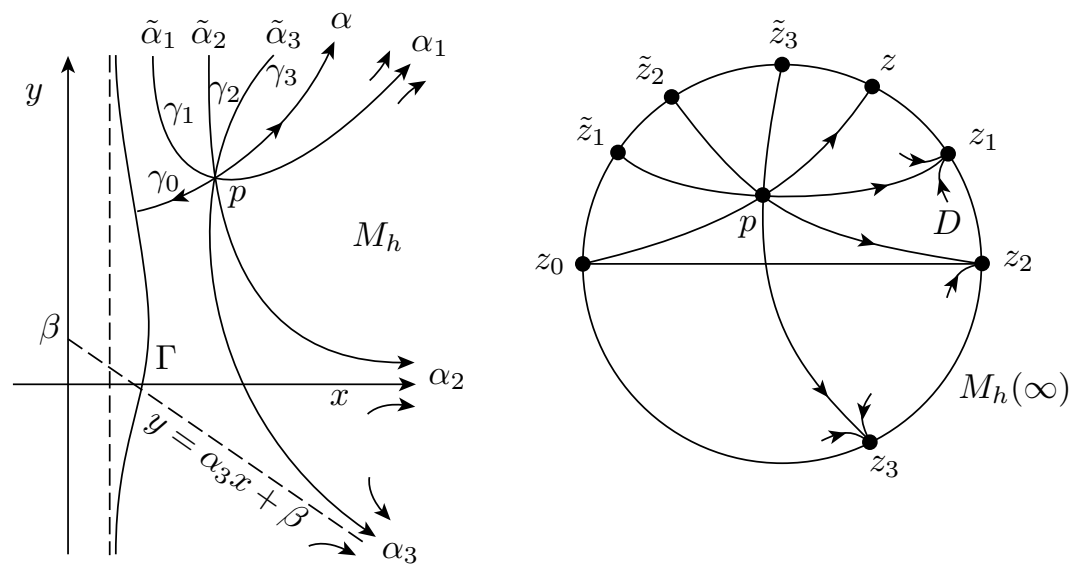

Figure 3. Geodesic flow for the repulsive isosceles problem.

\subsection{The Rhomboidal Coulombian Problem.}

We consider the problem obtained when we have 4 bodies with masses $m_{1}, m_{2}, m_{3}, m_{4}$ with Coulombian repulsive or gravitational attractive forces, having always a planar rhomboidal configuration (see $[\mathbf{L P C h}])$.

By using Jacobi-like coordinates with center of mass at the origin, if $x$ is the half distance between the particles of mass $m_{1}$ and $m_{2}$ with $m_{1}=m_{2}$ and $\frac{y}{A}$ is the half distance between the particles of mass $m_{3}$ and $m_{4}$ with $A=m_{3}=m_{4}$, the equations of motion are given by

$$
\left\{\begin{array}{c}
\ddot{x}=\frac{\gamma}{4 x^{2}}+\frac{2 \gamma A^{5 / 2} x}{\left(A x^{2}+y^{2}\right)^{3 / 2}} \\
\ddot{y}=\frac{\gamma A^{5 / 2}}{4 y^{2}}+\frac{2 \gamma A^{3 / 2} y}{\left(A x^{2}+y^{2}\right)^{3 / 2}}
\end{array}\right.
$$

where we suppose that $m_{1}=m_{2}=1$ (see Figure 4).

Here $\gamma$ is a parameter, where if $\gamma>0$ we have the Coulombian repulsive problem and if $\gamma<0$ we have the Celestial mechanics problem.

We observe that (9) can be studied as a Hamiltonian system whose energy function in the variables $x, y, \dot{x}, \dot{y}$ is written

$$
E=\frac{1}{2}\left[2 \dot{x}^{2}+2 \dot{y}^{2}\right]+\gamma\left(\frac{1}{2 x}+\frac{A^{5 / 2}}{2 y}+\frac{4 A^{3 / 2}}{\sqrt{A x^{2}+y^{2}}}\right)
$$


If we make the linear change of coordinates $u=\sqrt{2} x, v=\sqrt{2} y$ then the equations (9) become

$$
\left\{\begin{array}{l}
\ddot{u}=\frac{\sqrt{2}}{2} \frac{\gamma}{u^{2}}+\frac{4 \sqrt{2} A^{5 / 2} \gamma u}{\left(A u^{2}+v^{2}\right)^{3 / 2}}, \\
\ddot{v}=\frac{\sqrt{2}}{2} \frac{A^{5 / 2} \gamma}{v^{2}}+\frac{4 \sqrt{2} \gamma A^{3 / 2} v}{\left(A u^{2}+v^{2}\right)^{3 / 2}},
\end{array}\right.
$$

which has a Hamiltonian function in the variables $u, v, p_{u}, p_{v}$ given by

$$
\mathcal{H}=\frac{1}{2}\left[p_{u}^{2}+p_{v}^{2}\right]+U(u, v)
$$

where

$$
U(u, v)=\frac{\sqrt{2}}{2} \gamma\left[\frac{1}{u}+\frac{A^{5 / 2}}{v}+\frac{8 A^{3 / 2}}{\sqrt{A u^{2}+v^{2}}}\right] .
$$

By relabeling the variables, we can work with the Hamiltonian whose energy function in the variables $x, y, \dot{x}, \dot{y}$ has the form

$$
E=\frac{1}{2}\left[\dot{x}^{2}+\dot{y}^{2}\right]+\frac{\sqrt{2}}{2} \gamma\left[\frac{1}{x}+\frac{A^{5 / 2}}{y}+\frac{8 A^{3 / 2}}{\sqrt{A x^{2}+y^{2}}}\right] .
$$

We now restrict ourselves to the case $\gamma>0$ (Coulombian repulsive case). To estimate the curvature, we compute the Laplacian of the potential

$$
U(x, y)=\frac{\sqrt{2}}{2} \gamma\left[\frac{1}{x}+\frac{A^{5 / 2}}{y}+\frac{8 A^{3 / 2}}{\sqrt{A x^{2}+y^{2}}}\right]
$$

obtaining

$$
\Delta U=\frac{\sqrt{2}}{2} \gamma\left[\frac{2}{x^{3}}+\frac{2 A^{5 / 2}}{y^{3}}+\frac{8 A^{5 / 2} x^{2}(2 A-1)+8 A^{3 / 2} y^{2}(2-A)}{\left(A x^{2}+y^{2}\right)^{5 / 2}}\right] .
$$

And therefore, for $\Delta U \geq 0$ is sufficient that $2 A-1 \geq 0$ and $2-A \geq 0$, because $x>0, y>0$. But such conditions are equivalent to $\frac{m_{1}}{2} \leq m_{3} \leq$ $2 m_{1}$.

On the other hand, the potential (11) is homogeneous of degree -1 and it is easy to verify that is bounded at infinity.

Since the Hill's region in this case is connected and simply connected, and the connected boundary curve $\Gamma=\{U(x, y)=h\}$ has two limiting directions $z_{\alpha}=0$ and $z_{\beta}=\frac{\pi}{2}$ (see Figure 4 ), we obtain the 
Theorem 11 (Geometry of the Repulsive Rhomboidal Problem). If the masses satisfy the relation $\frac{m_{1}}{2} \leq m_{3} \leq 2 m_{1}$, we have that for a fixed energy level $h>0$, the set of physical curves is related, as a one-dimensional foliation, to the geodesic flow in a space homeomorphic to the unit disk with respect to a quasi-complete conformal metric with negative curvature (see Figure 4).

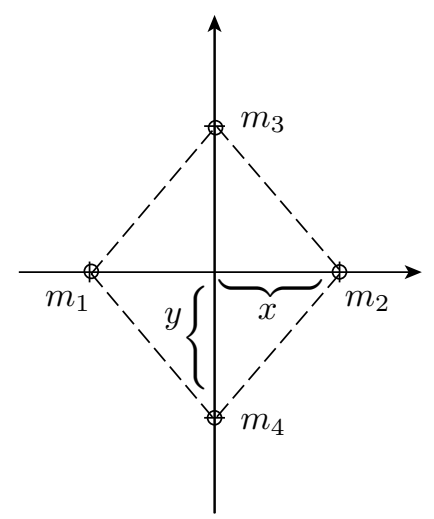

a) Describes the problem in Jacobi-like coordinates.
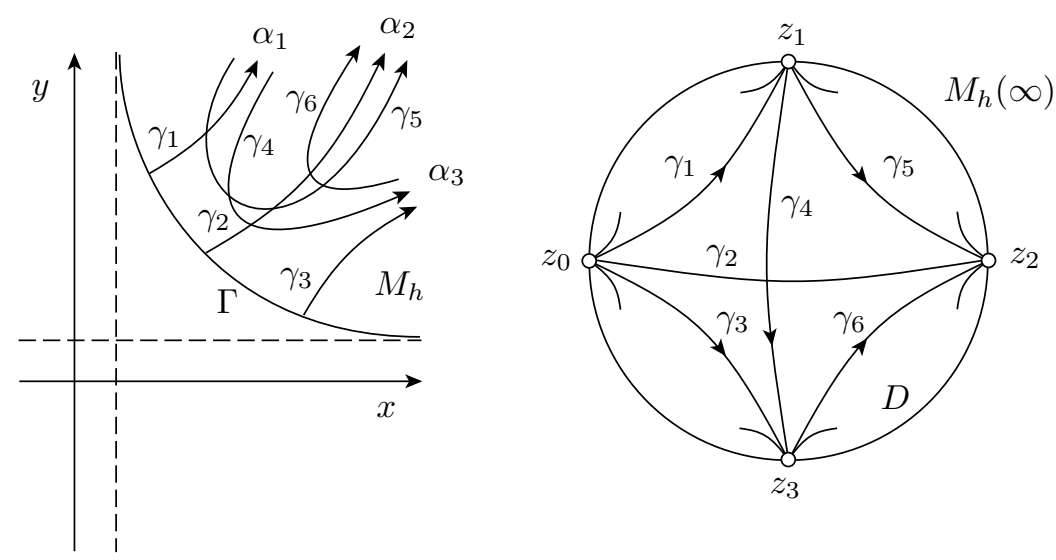

b) Illustrates Theorem 11 .

Figure 4. Geodesic flow for the rhomboidal repulsive problem. 


\section{References}

[A] D. I. Anosov And V. I. Arnold, "Dynamical Systems I," Springer-Verlag, Berlin, Heidelberg, 1988.

[Am] M. A. Armstrong, "Basic Topology," Mc Graw-Hill, London, New York, 1979.

[Ar] V. I. ARnold, "Mathematical Methods of Classical Mechanics," Springer-Verlag, New York, Heidelberg, Berlin, 1978.

[Du] B. Dubrovin, A. Fomenko and S. P. Novikov, "Geometrie Contemporaine, Methodes et Applications," $1^{\text {st }}$ part, Ed. MIR, Moscow, 1985.

[F] A. R. Forsyth, "Theory of Differential Equations," vol. III, Dover, 1900.

[Gr] M. Gromov, W. Ballman and V. Schroeder, "Manifolds of Nonpositive Curvature," Birkhäuser, Boston, Basel, Stuttgart, 1985.

[Kl] W. Klingenberg, "Riemannian Geometry," Walter de Gruyter ed., Berlin, New York, 1982.

[L1] E. A. Lacomba, Blow up on energy levels in celestial mechanics, Publ. Mat. 28 (1984), 97-117.

[L2] E. A. LACOMBA, Infinity manifolds for positive energy in celestial mechanics, Contemp. Math. 58, Part III (1987), 193-201.

[LBI] E. A. Lacomba, J. Bryant and L. A. Ibort, Blow up of mechanical systems with a homogeneous energy, Publ. Mat. 35 (1991), 333-345.

[LPCh] E. A. Lacomba and E. A. Pérez-Chavela, A compact model for the rhomboidal planar four-body problem, Celestial Mech. Dynam. Astronom. 54 (1992), 343-355.

[LR1] E. A. Lacomba and J. G. Reyes, Parallel escapes in the restricted repulsive Coulombian isosceles 3-body problem, in "New trends for hamiltonian systems and celestial mechanics," Advanced series in nonlinear dynamics 8, Singapore, World Scientific, 1996, pp. 260-273.

[LR2] E. A. Lacomba and J. G. Reyes, Unbounded Parallel and asymptotic solutions in the isosceles 3-body problems with inverse-square forces, Report no. 130, Centre de Recerca Matemàtica, Bellaterra, Barcelona, Spain (1991).

[O] C. P. Ong, Curvature and mechanics, Adv. Math. 15 (1975), 269-311. 
[Sp] M. A. SPIVAK, "A Comprehensive Introduction to Differential Geometry," vol. 1, Publish or Perish, Inc., Boston, Massachusetts, 1970.

\author{
Departamento de Matemáticas \\ Universidad Autónoma Metropolitana-Iztapalapa \\ Apartado postal 55-534 \\ 09340 México \\ D.F. MÉXICO \\ e-mail: lace@xanum.uam.mx \\ e-mail: revg@xanum.uam.mx
}

Primera versió rebuda el 8 de gener de 1997 , darrera versió rebuda el 10 de desembre de 1997 